\title{
LA IMPORTANCIA DE LAS VARIABLES MORFOLÓGICAS, MÉTRICAS Y DE MICRODESGASTE PARA EVALUAR LAS DIFERENCIAS EN DISEÑOS DE PUNTAS DE PROYECTIL BIFACIALES PEDUNCULADAS: UN EJEMPLO DEL SUR DE PATAGONIA CONTINENTAL.
}

\author{
NORA V. FRANCO ${ }^{1}$, ALICIA CASTRO ${ }^{2}$, MARCELO CARDILLO ${ }^{3}$ Y JUDITH CHARLIN $^{4}$
}

\begin{abstract}
RESUMEN
En este trabajo nos proponemos evaluar la existencia de variaciones en el diseño de puntas de proyectil bifaciales pedunculadas entre la cuenca del río Santa Cruz y el estrecho de Magallanes. Con este objetivo se aplicaron análisis morfométricos, métricos y de microdesgaste en los pedúnculos de estos instrumentos. Los mismos señalaron, por un lado, la existencia de semejanzas en su forma y por el otro, variaciones métricas y en el microdesgaste entre los conjuntos procedentes del norte y el sur del río Santa Cruz. Se discute si las causas de estas variaciones pueden relacionarse con características ambientales -como la disponibilidad de recursos líticos, faunísticos y vegetales- o con la existencia de diferentes poblaciones humanas.
\end{abstract}

PALABRAS CLAVES: Patagonia, puntas de proyectil bifaciales pedunculadas, análisis morfométricos, análisis métricos, análisis de microdesgaste.

\section{THE VALUE OF MORPHOLOGICAL, METRICAL AND MICROWEAR VARIABLES IN ORDER \\ TO EVALUATE DIFFERENCES IN THE DESIGN OF BIFACIAL STEMMED PROJECTILE POINTS: AN EXAMPLE FROM THE SOUTH OF CONTINENTAL PATAGONIA.}

\section{ABSTRACT}

The purpose of this paper is to evaluate the existence of variations in the design of stemmed bifacial projectile points recovered between the Santa Cruz basin and the Magellan Strait. Morphometric, metric and microwear analisys were applied on the stem of the proyectil points. While there are similar

1 CONICET - Universidad de Buenos Aries. Saavedra 15, 5to. piso, Ciudad Autónoma de Buenos Aires (C.P. 1083), Argentina, e-mail: nvfranco2008@gmail.com

2 Universidad de La Plata. Paseo del Bosque S/N. La Plata (C.P. 1900), Pcia. Buenos Aires, Argentina, e-mail: acastro@museo. fcnym.unlp.edu.ar

3 CONICET. Saavedra 15, 5to. piso, Ciudad Autónoma de Buenos Aires (C.P. 1083), Argentina, e-mail: marcelo.cardillo@gmail. com

4 CONICET. Saavedra 15, 5to. piso, Ciudad Autónoma de Buenos Aires (C. P. 1083), Argentina, e-mail: judith_charlin@yahoo. com.ar 
forms in the whole area, there are metrical and microwear differences between the samples coming for the north and south of the Santa Cruz basin. The causes of these results are evaluated in relation to environmental characteristics -lithic, prey and vegetation availability- and the possibility of the existente of different human populations.

KEYWORDS: Patagonia, stemmed bifacial projectile points, morphometric analysis, metric analysis, microwear analysis.

\section{INTRODUCCIÓN}

Las puntas de proyectil bifaciales pedunculadas tienen una amplia distribución en el sur de Patagonia continental. En este trabajo nos proponemos evaluar la potencialidad de diferentes análisis para dar cuenta de variaciones en el diseño de las mismas, identificar si hay una correlación entre las diferencias en el diseño y su distribución espacial y analizar si esas variaciones podrían relacionarse con la disponibilidad de diferentes recursos (rocas, fauna y vegetación) o con la presencia de diferentes grupos humanos.

\section{ANTECEDENTES}

Junius Bird postuló la presencia de diferencias diacrónicas en los diseños de puntas de proyectil bifaciales del sur de Patagonia desde los ca. 11.000 años AP (Bird 1938, 1946, 1988), reconociendo la existencia de puntas de proyectil bifaciales pedunculadas de tamaño mediano y pequeño, denominadas Fell IV y V, durante la última parte del Holoceno (Bird 1938, 1988) (Fig. 1 y 2).

Por otra parte, sobre la base de las semejanzas entre las puntas de proyectil etnográficas y arqueológicas, este investigador correlacionó el período Fell IV con los "Tehuelches" del sur de Patagonia y el Fell V con los "Onas" (Bird 1993). De esta manera, las puntas de proyectil Fell IV y V fueron consideradas como tipos diacrónicos sobre la base de su posición relativa. Posteriormente, este investigador obtuvo muestras para fechados radiocarbónicos, datando el período $\mathrm{V}$ en ca. 680 años AP en la cueva Fell (cf. Bird 1993, tabla 17). Bird dató por primera vez el período IV a partir de una muestra de carbón (fraccionada) obtenida de cañadón Cóndor, que aportó las fechas de 3.725 + 100 AP y 3.475 +/- 100 AP (Massone 1979). Posteriormente se obtuvieron fechas de $4.560+/$ -
130 AP y 4.280 +/- 50 AP. para el período IV en el sitio de laguna Tomas Gould (Massone 1981, Massone 1989-1990), obteniéndose cronologías de ca. 3.600 años AP para las denominadas puntas Fell V (Sanguinetti de Bórmida 1984). Además, Gomez Otero ha mostrado la co-existencia de ambos diseños después de ca. 2.000 años AP en el alero Potrok Aike, en el sur de la Patagonia argentina (Gomez Otero 1986-87, 1987, 1993), y se encontraron ambos diseños en asociación en ocupaciones del siglo XVI en Punta Dungeness 2, en proximidades del estrecho de Magallanes (Massone 1979). También al sur, en el campo volcánico Pali Aike, diferentes diseños de puntas de proyectil han sido recuperados en estratigrafía con posterioridad a ca. 3.500 años AP (Charlin 2009). Mientras tanto, al sur de la cuenca del río Santa Cruz estos diseños tienen cronologías más tardías comprendidas entre ca. 2.000 y 500 años AP (Franco 2002). Cabe señalar que Ratto (1994), a través del análisis de distintas variables de diseño -como la aerodinamia, la superficie de refuerzo y el tipo de enmangue de los cabezales líticos- intenta inferir su medio de propulsión, el
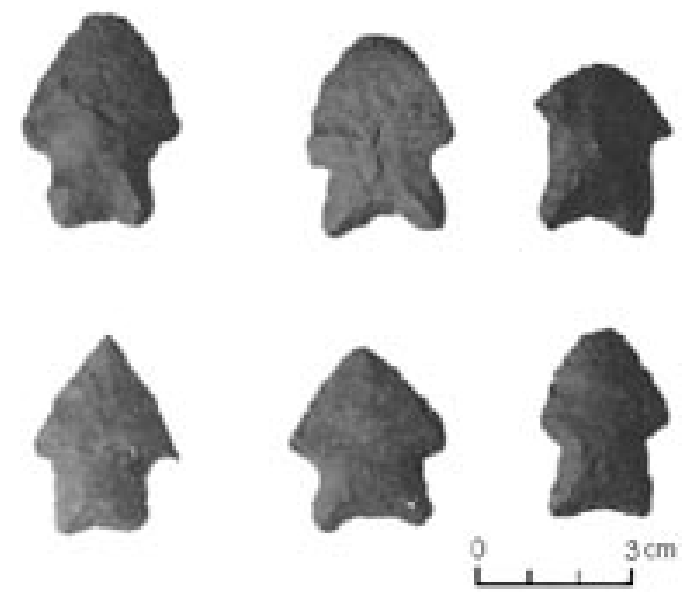

Fig. 1. Puntas de proyectil "Bird IV" (tomado de Bird 1993, figura 35, pág. 117). 


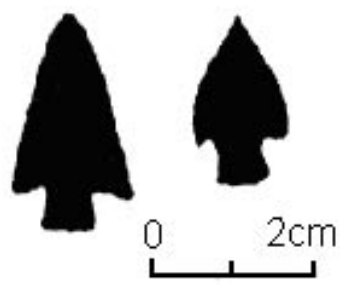

Fig. 2. Puntas de proyectil "Bird V" (tomado de Bird 1993, figura 34, pág. 116).

cual condiciona la implementación de técnicas de caza diferentes. De esta manera, esta investigadora propone que los cabezales líticos conocidos como Bird IV y V son producto de diferentes estrategias de caza, que coexisten en tiempo y espacio para el Holoceno tardío. Por consiguiente sus diferencias no son cronológico-culturales sino tan sólo funcionales (Ratto op. cit.). Esta autora postula la existencia de tres sistemas técnicos -lanzas arrojadizas propulsadas por fuerza muscular, flechas enastiladas propulsadas por arco y un arma de mano no arrojadiza- que coexisten en la cuenca de los ríos Gallegos y Chico, por lo menos entre los 3.600 y 740 años AP, según los fechados de los sitios considerados en su estudio. A diferencia de Prieto (1989-1990), quien descarta la posibilidad de que la reducción de tamaños que se observa entre los tipos Bird IV y V se encuentre relacionada con la introducción del arco y flecha en la Patagonia, Ratto sostiene que las puntas Bird V de El Volcán, las cuales fueron analizadas a partir de los datos publicados por Nami (1984a y b), corresponderían a flechas propulsadas por arco, en tanto que las Bird IV a lanzas arrojadizas. Para Prieto (1989-1999) las puntas tipo Bird V son de tamaño muy pequeño para ser efectivas a través de la caza con arco.

Cabe señalar además que, para el caso de las puntas denominadas Bird IV, Nami (1984 a y b, 1986, 2003) diferencia las etapas y técnicas de manufactura empleadas, teniendo en cuenta la formabase (nódulo o lasca) y el tamaño de la misma.

Las puntas de proyectil bifaciales pedunculadas consideradas en el presente análisis han sido encontradas tanto al norte como al sur del río Santa Cruz. Este río ha sido considerado como frontera entre diferentes poblaciones de cazadores-recolectores sobre la base de información etnográfica y de las características de los artefactos líticos -como la predominancia de rocas volcánicas oscuras, la baja frecuencia de tecnología de hojas y la mayor frecuencia de raederas por sobre raspadores al sur del río Santa Cruz-, y la frecuencia y características de los motivos rupestres -baja frecuencia, presencia casi única de la técnica de pintura y menor variedad de diseños de imagen al sur de esa cuenca- (ver por ejemplo, Vignati 1934; Orquera 1987; Fiore 2006). Algunas de estas variaciones podrían relacionarse con características ambientales, tales como la mayor disponibilidad de rocas de grano fino oscuras -mayoritariamente volcánicas- en el extremo sur de Patagonia continental (cf. Charlin 2009) o la menor frecuencia de rocas adecuadas para la confección de hojas al sur del río Santa Cruz (cf. Franco 2002). Otras evidencias sugieren, por otra parte, que el río Santa Cruz fue atravesado por las poblaciones humanas aún durante el Holoceno tardío. En este sentido apuntan los datos referidos a la obtención de obsidiana negra por parte de las poblaciones que ocupaban el sur del río Santa Cruz (cf. por ejemplo Franco 2002, 2004, 2008; Charlin 2008). Sin embargo, otras materias primas, como la obsidiana atigrada y la gris verdosa veteada -probablemente procedente de Sierra Baguales (entre otros Stern 2000)- tienen distribuciones diferenciales al norte y sur del río Santa Cruz (Franco et al. 2007; Franco 2008). Existen también formas diferentes de reactivación de raspadores en estos espacios, así como distintas formas de disposición de los muertos, al menos para algunos bloques temporales (Franco 2008). Creemos que las semejanzas y diferencias encontradas deben ser exploradas en mayor profundidad. Este trabajo es un paso en este sentido y se centralizará en algunas de las semejanzas y diferencias en los diseños de puntas de proyectil bifaciales pedunculadas recuperadas al norte y sur de este río.

Estudios previos -que incluyeron análisis estadísticos univariados de puntas de proyectil procedentes de superficie y excavación, además de muestras de museo recuperadas al sur de Patagonia continental y norte de Tierra del Fuego- sugieren la existencia de semejanzas métricas entre ellas, aunque pudieron reconocerse diferencias (Franco 1999; Franco y Borrero 2003). Los análisis multivariados efectuados sobre piezas de colecciones del Museo Británico -que fueron recuperadas en areas localizadas al norte y sur del río Santa Cruz, distantes más de 460 km en línea recta entre sí (Borrero y 
Franco 2001)-, muestran también la presencia de semejanzas en las variables métricas (Franco et al. 2005). Por otra parte, los análisis morfométricos aplicados a puntas de proyectil no fragmentadas procedentes de Patagonia continental señalan la existencia de una gran variabilidad en forma, relacionada en parte con la reactivación (Cardillo et al. 2005; Charlin y Cardillo 2009). La existencia de reactivación y la alta frecuencia de puntas de proyectil fragmentadas enfatiza la importancia de analizar las variaciones en el pedúnculo de estos ejemplares, por lo cual esta es la unidad de análisis considerada en el presente trabajo.

\section{MARCO TEÓRICO}

Las puntas de proyectil son sólo uno de los componentes de un sistema de armas aunque, desafortunadamente, en muchos casos son la única parte que perdura. Consideramos que las variaciones en diseño y otras características de los artefactos deben ser entendidos dentro del marco de la organización tecnológica (Nelson 1991). En este sentido, a los efectos de entender los cambios en la forma de los artefactos, es importante tener en cuenta la distribución de los recursos líticos, faunísticos y vegetales.

La gente desarrolla sus actividades de distintas maneras, es decir, eligen diferentes formas de hacer las mismas cosas, no sólo debido a las variaciones que existen en el ambiente, sino también al conocimiento generado por la transmisión vertical (generacional) y horizontal (entre coetáneos) de información (Cavalli Sforza y Feldman 1981). Sin embargo, los artefactos se incorporan al registro arqueológico después de un largo proceso, que frecuentemente involucra la reactivación, la cual en muchos casos puede llevar a la pérdida de la forma original de la pieza. Por esta razón, nuestro principal problema es la posibilidad de reconocer las elecciones hechas por los cazadores-recolectores, especialmente teniendo en cuenta los problemas de equifinalidad tan frecuentes en el registro arqueológico.

\section{PROCEDENCIA Y CARACTERÍSTICAS DE LA MUESTRA}

Este trabajo se focaliza en el área comprendida entre el norte de la cuenca del río Santa Cruz y el estrecho de Magallanes (fig. 3).

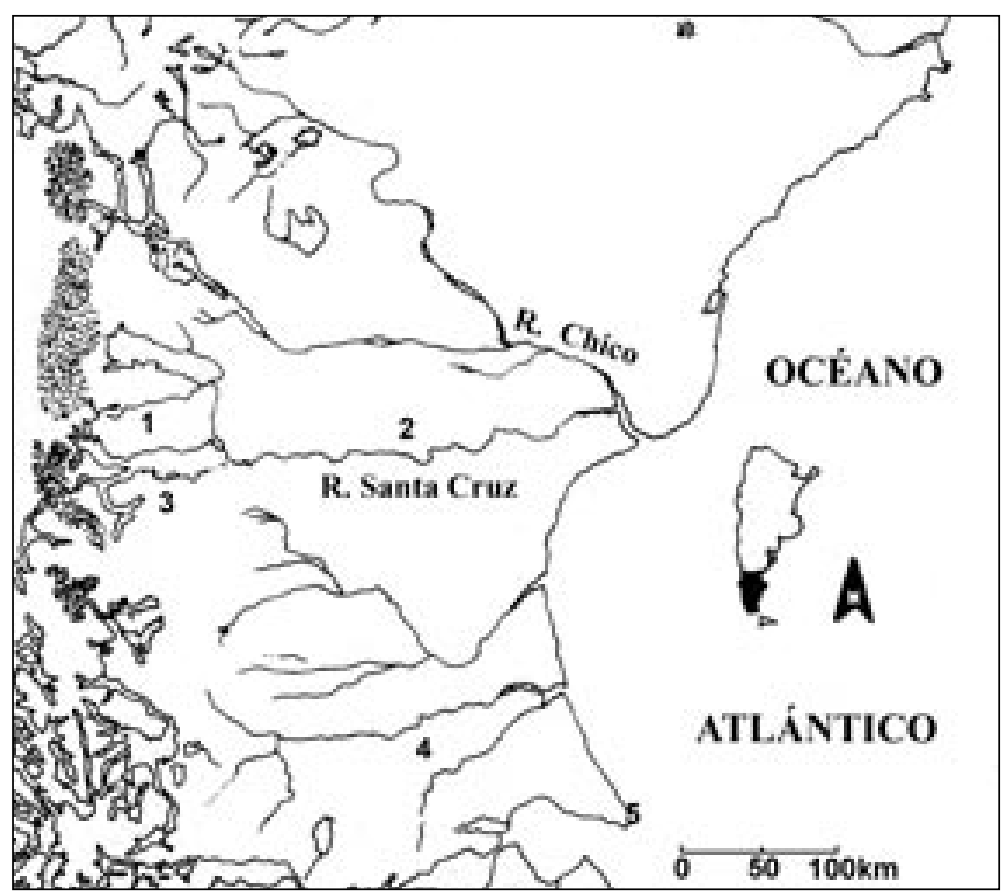

Fig. 3. Sur de Patagonia continental, con la procedencia de las muestras: 1) Norte del Lago Argentino; 2) Norte de la cuenca superior y media del río Santa Cruz, 3) Sur de Lago Argentino, 4) campo volcánico Pali Aike, 5) Cabo Vírgenes. 
Los conjuntos de puntas de proyectil bifaciales pedunculadas que componen la muestra bajo estudio son los siguientes (tabla 1 ):

Tabla 1. Puntas de proyectil bifaciales pedunculadas que componen la muestra.

\begin{tabular}{|c|c|c|}
\hline \multicolumn{2}{|c|}{ Procedencia } & Frecuencia \\
\hline \multirow{3}{*}{ NORTE } & Norte Lago Argentino & 12 \\
\cline { 2 - 3 } & $\begin{array}{c}\text { Norte curso superior y } \\
\text { medio de río Santa Cruz }\end{array}$ & 13 \\
\hline \multirow{3}{*}{ SUR } & Sur Lago Argentino & 7 \\
\cline { 2 - 3 } & Pali Aike & 24 \\
\cline { 2 - 3 } & Cabo Vírgenes & 7 \\
\hline
\end{tabular}

a) Norte de la cuenca del río Santa Cruz:

a.1.) Norte de Lago Argentino: la muestra incluye 12 artefactos recuperados tanto en cotas bajas (ca. $200 \mathrm{~m}$ ) como altas (ca. $850 \mathrm{~m}$ ), manufacturados en dacita, calcedonia y rocas de grano fino oscuras (sensu Charlin 2005). Ésta es un área con pocos aleros sobre soportes sedimentarios. Se indica con el número 1 en la figura 3.

a.2) Norte de la cuenca superior y media del río Santa Cruz: se incluyen 13 puntas de proyectil confeccionadas sobre dacita, obsidiana, jaspe, ópalo y rocas de grano fino oscuras. El área comprende espacios al aire libre y afloramientos volcánicos, en los que son frecuentes los aleros y cuevas sobre roca volcánica y minoritariamente sedimentaria (ver número 2 en figura 3). Las altitudes en este área oscilan entre ca. 200 y $1.000 \mathrm{msnm}$.

b) Sur de la cuenca del río Santa Cruz:

b.1) Sur de Lago Argentino: se trata de 7 artefactos, manufacturados sobre dacita y rocas de grano fino oscuras. El área posee escasos aleros, mayoritariamente en afloramientos volcánicos, con altitudes entre 200 y 1.100 msnm (número 3 en figura 3).

b.2) Campo volcánico Pali Aike: la muestra incluye 24 puntas de proyectil, mayoritariamente confeccionadas sobre rocas de grano fino oscuras, siendo en general basaltos (Charlin 2009). Hay también algunos ejemplares confeccionados sobre calcedonia y dacita. Se trata de un área de afloramientos volcánicos, con abundantes abrigos y cuevas en el sector sur (Corbella 2002; D’Orazio et al. 2000) y alturas que oscilan entre ca. 200 y 1000 msnm (número 4 en figura 3).

b.3) Cabo Vìrgenes: este conjunto comprende 7 artefactos, la mayoría sobre rocas de grano fino oscuras. Hay un único artefacto confeccionado sobre calcedonia. No hay aleros en el área, que está muy cercana al océano Atlántico y tiene alturas de ca. 200 msnm (número 5 en figura 3).

\section{METODOLOGÍA}

Se llevaron a cabo tres diferentes tipos de análisis: de morfometría geométrica, métrico y de microdesgaste.

a) Análisis de morfometría geométrica

Los análisis de forma basados en landmarks (Bookstein 1991) permiten realizar un análisis cuantitativo de la misma a partir de imágenes previamente digitalizadas (Rohlf 1990), independientemente del tamaño efectivo del objeto. Esto permite separar forma y tamaño y estudiarlo de manera independiente (Zelditch et al. 2004). Para los análisis morfométricos se emplearon 54 puntas de proyectil provenientes de las distintas áreas mencionadas. Para describir los contornos de los pedúnculos se colocaron diez puntos: dos landmarks o puntos de correspondencia geométrica ubicados en máximos de curvatura (Bookstein 1991) y ocho semilandmarks o puntos espaciados a intervalos regulares para describir el contorno comprendido entre los puntos antes mencionados (Bookstein 1997).

Con el fin de evitar la información en la señal morfológica debida a la asimetría de la forma, se utilizó sólo uno de los lados del pedúnculo. Los diez puntos fueron transformados en información morfológica mediante el método de procrustes y sometidos a análisis de componentes principales (relative warps) (Bookstein 1989) para obtener nuevas variables que contengan la mayor cantidad de información morfológica posible (Bookstein 1991; Rohlf 1993). Para realizar estos análisis se empleó el paquete estadístico TPS (Rohlf 2004).

Posteriormente, para explorar posibles diferencias en la morfología entre áreas se aplicó análisis discriminante utilizando como variables los dos primeros ejes de los componentes principales de forma (que comprenden el 75\% de la variación) mediante el programa Past (Hammer y Harper 2001). 


\section{b) Análisis métrico}

Para el análisis métrico se emplearon 66 casos, en los cuales se registraron las siguientes variables: largo, ancho y espesor del pedúnculo y espesor y ancho del cuello. Las medidas fueron tomadas en $\mathrm{mm}$ con calibre digital con una definición de dos decimales. Al igual que en el caso morfométrico, el análisis discriminante fue empleado para explorar diferencias entre sectores a partir de los resultados de los componentes principales.

\section{c) Análisis de microdesgaste}

A los efectos de analizar las variaciones en los pedúnculos, se hicieron también análisis de microdesgaste. Entendemos que los mismos pueden proporcionar información sobre la forma de enmangue. A este respecto, cabe señalar que tanto trabajos realizados por otros autores (Anderson et al. 1993; Beyries 1987; Rots 2003, 2005) como test ciegos realizados por uno de nosotros (Castro y Moreno 1994) permitieron reconocer que, al menos en algunos casos, es posible identificar patrones de microdesgaste relacionados con la existencia de distintas formas de enmangue en artefactos líticos de distinto tipo y sobre diferentes materias primas. Estos microdesgastes se producirían por contactos con las diferentes sustancias que conforman la estructura del astil y presiones mínimas de uso, que dejarían algún tipo de huella (cf. Cahen, Keeley y Van Noten 1979; Vaughan 1985). La generación de estos patrones se espera que sea mayor cuanto más larga sea la duración de la vida útil del instrumento o más intensa la fricción. Cabe señalar también que los rastros podrían generarse no sólo durante el impacto, sino también a lo largo de la elaboración, mantenimiento y transporte de los proyectiles. Por otra parte, no se descarta que, en algunos casos de reclamación, la misma forma de enmangue pueda haberse mantenido.

Sobre la base de las características microcristalinas de las materias primas en las que fueron confeccionadas las puntas de proyectil y las observaciones de su aspecto microsuperficial, inferimos la factibilidad de desarrollo de micropulidos diagnósticos sobre diferentes sustancias de contacto identificables y descriptibles según las metodologías aplicadas al estudio de sílices europeos (entre otros Keeley 1980; Anderson 1981). Experiencias sobre distintas materias primas, especialmente de Patagonia centromeridional (Mansur 1999; Alvarez 2004; Castro 1989; Reyes y Castro 2004; Martucci y Castro en prep.) nos permiten confiar en el diagnóstico empírico comparativo-cualitativo, que podríamos calificar como subjetivo (Keeley 1980; Castro 1996), en contraposición con otras metodologías de aproximación objetiva (Toselli et al 2005; Alvarez 2002; Ibañez Estévez y Gonzalez Urquijo 2000).

Los análisis se realizaron directamente sobre los pedúnculos de 44 piezas, observando las superficies centrales de ambas caras y los filos de las aristas laterales y la base. En este sentido, cabe señalar que el patrón de enmangue se infirió sobre la base del análisis del pedúnculo en forma directa. No se utilizó en este caso, el análisis indirecto, tal como fue efectuado por Mansur (1987), quien tuvo en cuenta variaciones en otras partes de la morfología funcional de la pieza para inferior si la misma fue enmangada.

Los análisis de bajos y altos aumentos fueron llevados a cabo utilizando una lupa triocular estereoscópica con aumento de 50X, básicamente para analizar alteraciones morfológicas en filos y aristas, brillos y desgastes. Se utilizó también un microscopio metalográfico de luz incidental marca UNION con aumentos de 280X para identificar estrías, redondeamiento de aristas y micropulidos.

En este caso y a los efectos de alcanzar los objetivos del trabajo, pusimos énfasis en las siguientes variables y en el interjuego de combinaciones entre ellas:

1- desarrollo de microdesgaste (o micropulidos), estrías y daño morfológico del filo: estas variables se consideran diagnósticas de contacto. Por otra parte y en particular las estrías, son indicadoras de orientación o dirección de movimiento. El daño morfológico del filo, no encontrado en partes morfológicas funcionales, sugiere alteraciones producidas por otros fenómenos.

2- distribución del microdesgaste y daños en relación con filos y caras (unifacial o bifacial y marginal o extendido): La posición relativa de las microalteraciones -aún cuando no sean diagnósticas- combinadas con los daños morfológicos del filo, permiten discutir situaciones particulares de contacto (entre otros, Tringham et al. 1974; Castro 1994).

3- intensidad: permite discutir material o sustancia y forma de contacto. 
Los resultados nos llevaron a identificar distintos tipos de microalteraciones y diferentes formas de distribucion de los mismos en las partes observadas y concluir que pueden relacionarse con tres patrones de enmangue distintos (cf. infra).

\section{RESULTADOS}

a) Análisis morfométrico

$\mathrm{El}$ análisis de relative warps (RW) muestra que los dos componentes principales explican el $75 \%$ de la variación morfológica observada (RW1 49\%, RW2 25\%). El primer eje (RW1) señala que la mayor variación corresponde a cambios en la base del pedúnculo (los puntajes positivos del RW1 muestran una base más expandida y cóncava, mientras que los puntajes negativos se corresponden con bases rectas y menos cóncavas). El RW2 muestra la expansión y contracción relativa del pedúnculo (figura 4 y tabla 2).

El análisis discriminante sobre los dos componentes de forma señala que no hay diferencias significativas entre los sectores del espacio considerados (Hotelling $t^{2} p=0.50$, porcentaje correctamente clasificado a uno u otro sector: 59,26\%).
Tabla 2. Valores singulares y porcentaje explicado por los Relative Warps.

\begin{tabular}{|c|c|c|c|}
\hline No. & Valor Singular & $\%$ & $\%$ Acumulado \\
\hline 1 & 0.57586 & $48,97 \%$ & $48,97 \%$ \\
\hline 2 & 0.40795 & $24,58 \%$ & $73,54 \%$ \\
\hline 3 & 0.27516 & $11,18 \%$ & $84,72 \%$ \\
\hline 4 & 0.22136 & $7,24 \%$ & $91,96 \%$ \\
\hline 5 & 0.12632 & $2,36 \%$ & $94,32 \%$ \\
\hline 6 & 0.11228 & $1,86 \%$ & $96,18 \%$ \\
\hline 7 & 0.09070 & $1,21 \%$ & $97,39 \%$ \\
\hline 8 & 0.07572 & $0,85 \%$ & $98,24 \%$ \\
\hline 9 & 0.05564 & $0,46 \%$ & $98,70 \%$ \\
\hline 10 & 0.04997 & $0,37 \%$ & $99,06 \%$ \\
\hline 11 & 0.04701 & $0,33 \%$ & $99,39 \%$ \\
\hline 12 & 0.03995 & $0,24 \%$ & $99,63 \%$ \\
\hline 13 & 0.03469 & $0,18 \%$ & $99,80 \%$ \\
\hline 14 & 0.02526 & $0,09 \%$ & $99,90 \%$ \\
\hline 15 & 0.01911 & $0,05 \%$ & $99,95 \%$ \\
\hline 16 & 0.01808 & $0,05 \%$ & $100,00 \%$ \\
\hline
\end{tabular}

b) Análisis métrico

El análisis de componentes principales fue realizado a partir de una matriz de correlación con el fin de estandarizar la muestra y reducir el peso de la diferencia en la escala. Los dos primeros ejes explican el 97\% de la variación en tamaño (CP1=93,43\%) e

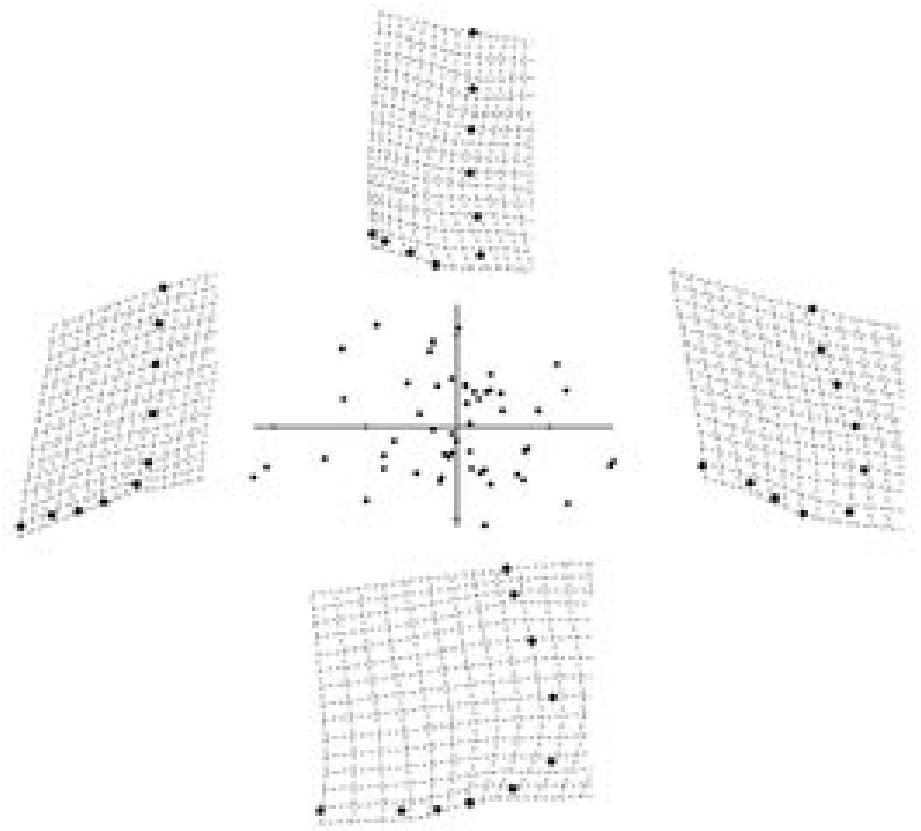

Fig. 4. Principales tendencias de variación morfológica en la muestra (grillas de deformación correspondientes a los dos primeros Relative Warps). 
$N$

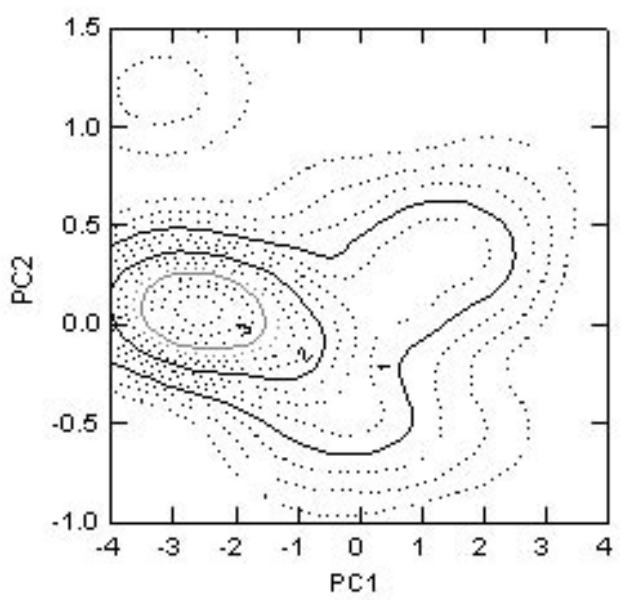

$S$

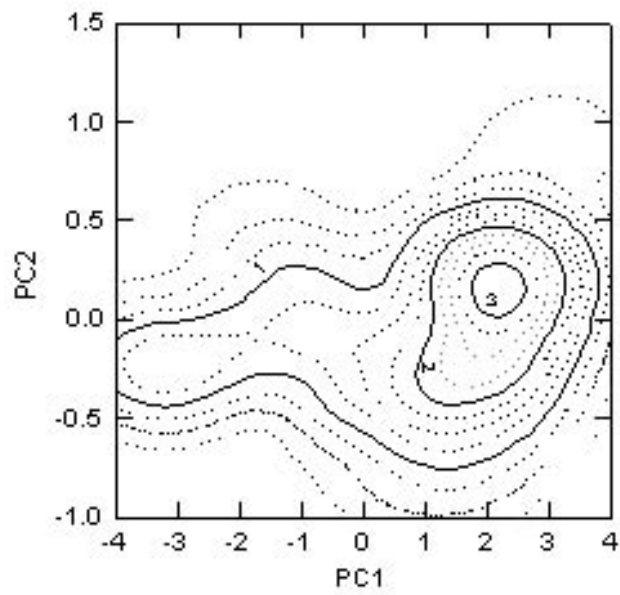

Fig. 5. Estimación de densidad de Kernel para los dos primeros componentes principales.

indican que todas las variables tienen peso similar en el patrón observado en este componente. Los resultados sugieren que los ejemplares provenientes del sur del río Santa Cruz son más largos, anchos y espesos.

El análisis discriminante sobre los dos componentes principales señala que existen diferencias significativas entre las variables de tamaño entre ambos grupos (Hotelling $t 2 p=0.0005$, porcentaje correctamente clasificado: $71,21 \%)$.

El gráfico de los dos componentes principales utilizando la estimación de densidad de Kernel (figura 5) muestra la diferencia en la frecuencia de tamaño entre ambas áreas. Cabe señalar que existen diferencias en las materias primas utilizadas para la manufactura de puntas de proyectil bifaciales

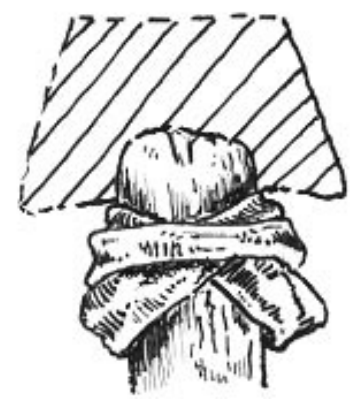

Fig. 6. Enmangue cruzado. pedunculadas al norte y sur de la cuenca, siendo más frecuentes las rocas de grano fino oscuras al sur. Esta diferencia es estadísticamente significativa $\left(C h i^{2}=87,33 \mathrm{p}=0.000\right)$ y está en relación con la disponibilidad de rocas a escala supra-regional.

En general, los resultados muestran que las puntas de proyectil bifaciales pedunculadas procedentes del sur del río Santa Cruz son en promedio más anchas, largas y espesas que las recuperadas al norte del área.

c) Análisis de microdesgaste

Se encontraron huellas sólo en 39 ejemplares. En algunos casos, el tipo de enmangue no pudo identificarse. Los resultados obtenidos en 28

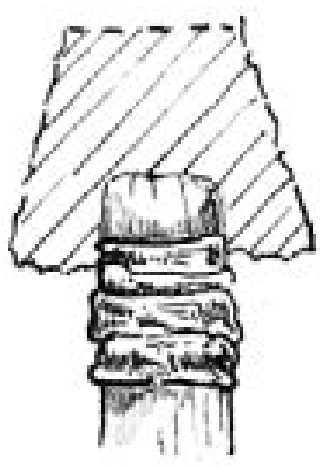

Fig. 7. Enmangue recto. 
muestras indican la presencia de tres diferentes tipos de enmangue, que pueden ser relacionados con diferentes métodos de prehensión:

I) En algunos casos el micropulido puede relacionarse con la existencia de cuero o piel sobre el pedúnculo, tanto cerca del cuello (o raíz) como de la parte distal del mismo. Este micropulido de cuero presenta una orientación oblicua, asociado a estrías con la misma dirección en las saliencias laterales de la base de los pedúnculos. La parte central de las caras del pedúnculo presenta brillo de madera, que puede probablemente relacionarse con el mango. La figura 6 muestra nuestra interpretación de este tipo de enmangue, que hemos denominado "cruzado". Se considera que, en este caso, el cuero ha envuelto incluso las aletas salientes del pedúnculo.

II) En otros casos, se observó micropulido de madera en el cuello y también en algunos casos en el pedúnculo. Éste se presenta en relación con un micropulido de cuero o piel desarrollado a lo largo de las aristas laterales del pedúnculo, asociado en algunas oportunidades a estrías transversales. Hemos denominado a este enmangue "recto" (figura 7).

III) Algunas piezas sólo presentan un micropulido que puede relacionarse con cuero en todo el cuerpo del pedúnculo. Hemos denominado a este tipo de enmangue "pedúnculo cubierto en cuero".

La tabla 3 muestra los distintos tipos de enmangue identificados y la procedencia de las muestras:

Tabla 3. Tipos de enmangue y procedencia de las muestras.

\begin{tabular}{|c|c|c|}
\hline Tipo de enmangue & $\begin{array}{c}\text { Norte del río } \\
\text { Santa Cruz }\end{array}$ & $\begin{array}{c}\text { Sur del río } \\
\text { Santa Cruz }\end{array}$ \\
\hline Enmangue recto & 3 & 11 \\
\hline Enmangue de cuero & 5 & 3 \\
\hline Enmangue cruzado & 0 & 6 \\
\hline Total & 8 & 20 \\
\hline
\end{tabular}

Se observaron diferencias significativas entre la frecuencia de tipo de enmangue entre los sectores norte y sur del río Santa Cruz $\left(\mathrm{Chi}^{2}=62.43, \mathrm{p}=\right.$ 0.001). Esta diferencia parece deberse básicamente a que el enmangue cruzado sólo se ha identificado, hasta el momento, al sur del río Santa Cruz.

d) Otros resultados

Sobre la base de las diferencias previamente observadas, se llevó a cabo el análisis de la varianza
(ANOVA) de un factor (Sokal y Rolhf 1979) entre las distintas variables métricas y los resultádos del análisis microscópico. Las diferencias entre la cantidad de casos fueron ajustadas mediante el test de Bonferroni, para corregir el nivel de $p$ en cada caso. Ya que las variables métricas tenían una distribución normal, no fue necesario en ningún caso el tratamiento previo de los datos.

El análisis de varianza señala que existe una relación no aleatoria entre el enmangue (la variable independiente) y algunas de las variables métricas. Se observaron diferencias estadísticamente significativas ( $F=4.69, p=0.02)$ entre el largo del pedúnculo cubierto en cuero (media $=8.48 \mathrm{~mm}$ ) y el cruzado (media $=15.62 \mathrm{~mm}$ ). Asimismo, hay diferencias entre el espesor del pedúnculo y el ancho del cuello en relación con estos dos tipos de enmangue. El espesor medio para el enmangue en cuero del pedúnculo es de $3.86 \mathrm{~mm}$, mientras que el del enmangue cruzado es $6.93 \mathrm{~mm}(\mathrm{~F}=4.24, p=0.02)$. El ancho medio del cuello es $4.12 \mathrm{~mm}$ para los pedúnculos envueltos en cuero y $7.39 \mathrm{~mm}$ para los de enmangue cruzado $(\mathrm{F}=5.42, p=0.01)$.

\section{DISCUSIÓN}

En resumen, de acuerdo con el análisis morfométrico, no hay diferencia en la forma media entre las puntas de proyectil recuperadas al norte y sur de la cuenca del río Santa Cruz. Esto significa que se encuentran morfologías similares en toda el área. Por el contrario, los análisis métricos y de microdesgaste sugieren la existencia de diferencias entre los artefactos recuperados al norte y sur del río Santa Cruz. Los análisis macroscópicos que tienen en cuenta las diferencias en el espesor del pedúnculo y limbo de las puntas de proyectil indican también que, al menos algunas de las muestras recuperadas al sur de la cuenca fueron reactivadas mientras estaban enmangadas (Franco 1999), sugiriendo que tuvieron una larga vida útil. Los análisis preliminares métricos y morfométricos sugieren también cambios en la morfología del limbo que pueden deberse al proceso de reactivación (Cardillo obs. pers.; Charlin 2009; Franco 1999). Los análisis de microdesgaste del limbo llevados a cabo por Alicia Castro indican, por otra parte, que antes de ser descartadas, algunas puntas fueron utilizadas para cortar, raspar y perforar. 
¿Cuáles son, en síntesis, las diferencias que se encuentran entre las puntas de proyectil bifaciales pedunculadas procedentes del norte y sur del río Santa Cruz? Como ya mencionamos, los análisis multivariados realizados sobre variables métricas indican que las puntas recuperadas al sur son más largas, anchas y espesas que las procedentes del norte. El análisis discriminante realizado sobre los dos primeros componentes principales indica que hay diferencias significativas en el tamaño entre sectores, con un $71 \%$ de éxito en la asignación de pertenencia a uno u otro grupo a partir del mismo. En lo que respecta al análisis microscópico, hay diferencias en la frecuencia de distintos tipos de enmangue entre áreas, así como una relación entre el tipo de enmangue y algunas variables métricas. También hay diferencias estadísticas entre el tamaño promedio de la longitud, ancho y espesor en el pedúnculo en el caso del pedúnculo con enmangue cruzado y el envuelto en cuero. El enmangue recto tiene valores intermedios.

¿Cómo podemos explicar esas diferencias? Primero, podemos decir que las variaciones no se relacionan con la disponibilidad de presas, ya que el guanaco ha sido la principal fuente de alimento durante todo el Holoceno para las áreas analizadas (entre otros Borrero 1994-95; Mengoni Goñalons 1999). Como mencionamos, Ratto (1993, 1994) considera que algunas de las diferencias entre diseños pueden relacionarse con la existencia de diferentes sistemas de armas. Los datos experimentales y etnográficos enfatizan la importancia del ancho del pedúnculo para diferenciar dardos y flechas (por ejemplo Odell y Cowan 1986; Howard 1974; Ratto 1988), considerando que un diámetro de $10 \mathrm{~mm}$ es una buena estimación de la división entre ambos tipos de sistemas de armas (Ratto 1994).

Con el fin de dar cuenta de posibles diferencias en tamaño relacionadas con sistemas técnicos se repitieron los tests removiendo los casos con anchos de cuello menores a los $10 \mathrm{~mm}$, obteniéndose resultados similares a los ya indicados. En síntesis, algunas de las variaciones en diseño podrían relacionarse con la existencia de diferentes sistemas de armas (por ejemplo, puntas de dardo versus puntas de flecha). Sin embargo, hay también algunas variaciones que no pueden ser explicadas por esta razón. ¿Cuáles son las causas posibles de estas variaciones?
Hemos mencionado que existen diferencias en las rocas empleadas para la confección de puntas entre sectores, la que se encontraría relacionada con la disponibilidad de materias primas en cada uno de estos espacios. ¿Pueden las diferencias en el tamaño de los nódulos disponibles en estos sectores explicar las variaciones encontradas? Creemos que no es el caso, ya que a pesar de que los nódulos de rocas de grano fino oscuras son más pequeños que los de dacita al sur del río Santa Cruz, los ejemplares procedentes de este sector del espacio son los más grandes. Esto sugiere que las variaciones encontradas no están en relación con el tamaño de los nódulos lo que, sin embargo, debe ser explorado en mayor detalle. Resta analizar, además, si la tenacidad o dureza de las rocas disponibles pueden ser la razón para las diferencias encontradas.

¿Qué podemos decir de la disponibilidad de madera? En general, es la misma en ambas áreas. El Nothofagus -que proporciona las maderas más largas- está localizado en ambos casos al oeste de este espacio desde al menos 4.000 años AP en algunas de las áreas estudiadas (Mancini 2002, Mancini et al. 2005). El calafate (Berberis sp.) que, de acuerdo con la información etnográfica (Childs 1997 /1936/), ha sido utilizado para la manufactura de arcos, está disponible en ambas áreas en la estepa (entre otros Mancini 2002). La principal diferencia en la disponibilidad de vegetales entre el norte y sur de la cuenca del río Santa Cruz podría ser la mayor abundancia de molle (Schinus johnstonii) al norte (Mancini com. pers.). El molle produce un tipo de resina que podría haber sido utilizado para el enmangue de puntas de proyectil. Por lo tanto, la menor disponibilidad de resinas al sur podría haber enfatizado la necesidad de mantener la punta de proyectil dentro del mango, extendiendo su vida útil. El refuerzo del pedúnculo al sur sería consistente con este hecho. Sin embargo, Azorella sp., que podría haber sido utilizada también como resina, está disponible en ambas áreas (Rapoport et al. 2003).

Otros factores que podrían explicar las diferencias encontradas son:

a) la presencia de grupos humanos que en su movilidad abarcan diferentes sectores del espacio -el norte y el sur de la cuenca del río Santa Cruz, respectivamente- durante la última parte del Holoceno. Las semejanzas en morfología con di- 
ferencias en los tamaños podrían explicarse como el resultado de diferentes formas de manufactura de las puntas de proyectil (entre otros Close 1978; Wiessner 1983), las que deberán ser analizadas en detalle. Las semejanzas, en este caso, podrían ser el resultado del intercambio de información entre ambas áreas. Esta hipótesis es consistente con las evidencias de intercambio de obsidiana entre el norte y sur de la cuenca del río Santa Cruz con posterioridad a ca. 3.000 años AP (Charlin 2009; Franco 2002, 2008).

b) diferencias cronológicas entre las muestras. La escasa cantidad de muestras en estratigrafía no permite aún controlar este factor.

Podemos decir entonces que los análisis que resultaron más útiles hasta el momento para diferenciar entre distintos diseños de puntas de proyectil bifaciales pedunculadas del extremo sur de Patagonia son los métricos y los de microdesgaste. Los análisis morfométricos sirvieron para indicar la existencia de cambios en la morfología de estos artefactos en relación con la reactivación, que complementan los ya obtenidos con otras metodologías. Resta ahora entender la razón de las diferencias observadas. En este sentido nuestros siguientes pasos serán: 1) ampliar el número de muestras para el análisis, incluyendo puntas de proyectil con información cronológica, 2) analizar la tenacidad y dureza de las rocas a efectos de explicar las diferencias en diseño, 3) hacer análisis de residuos en puntas de proyectil de manera de evaluar el efecto de la disponibilidad de resinas, 4) incluir datos adicionales morfológicos, métricos y tecnológicos en el análisis.

Creemos que esta clase de análisis, así como aquellos que pueden ser llevados a cabo utilizando otros instrumentos u otras líneas de evidencias -como los isótopos estables y la distribución y características de motivos y técnicas rupestres- pueden ser útiles para entender la movilidad humana en áreas amplias.

\section{AGRADECIMIENTOS}

A los evaluadores y a Luis Borrero por la lectura crítica de este manuscrito. Esta investigación fue financiada por la Agencia Nacional de Promoción Científica y Técnica (PICT 19-26040 y 04-9498), la Universidad de Buenos Aires (UBACyT F140, F119 y F133), el CONICET (PIP 5209, 2390 y
5676) y National Geographic Society (Grant 773604). Queremos agradecer a las Estancias Chorrillo Malo, Anita, La Laurita, La Meseta, Bi Aike, María Elena, Condor Cliff, La Barrancosa, Yaten Guajen, Marta, El Tranquilo, Monte Entrance, Luz Divina, El Sosiego, La Querencia, La Angostura, Irene, Cóndor, Don Bosco y Tres de Enero.

\section{BIBLIOGRAFÍA}

ÁLVAREZ, M. R. 2003. Organización tecnológica en el Canal Beagle. El caso de Túnel I (Tierra del Fuego, Argentina). Tesis doctoral. Facultad de Filosofía y Letras, Universidad de Buenos Aires. Buenos Aires.

- 2004. El uso de materias primas vegetales en la costa norte del canal Beagle a través del análisis funcional de base microscópica. En: Contra viento y marea. Arqueología de la Patagonia, pp. 279-294. Comps. M. T. Civalero, P. M. Fernandez y A. G. Guráieb. Instituto Nacional de Antropología y Pensamiento Latinoamericano y Sociedad Argentina de Antropología. Buenos Aires.

ANDERSON, G. 1981. Contribution méthodologique a l'analyse des micro-traces d'utilization sur les outils préhistoriques. Tesis de Doctorado. Universite de Bordeaux 1. Bordeaux

ANDERSON, P.; S. BEYRIES, M. OTTE y H. PLISSON (ed.). 1993. Traces et function: les gestes retrouvés. ERAUL 50. Lieja

BEYRIES, S. 1987. Quelques exemples de stigmates d'emmanchements observés sur des outils du Paléolithique moyen. La main et l'outil: manches et emmanchements préhistoriques. Travaux de la Maison de l'Orient 15:55-62.

BIRD, J. 1938. Antiquity and migrations of the early inhabitans of Patagonia. Geographical Review XXVIII: 250-275.

BIRD, J. 1946. The Archaeology of Patagonia. En: Handbook of South American Indians, Steward, J. H. (Ed.), Smithsonian Institution, Bureau of American Ethnology, vol. 1, pp. 17-24. Washington.

BIRD, J. 1988. Travels and Archaeology in South Chile, Hyslop, J. (Ed.). University of Iowa Press, Iowa.

BOOKSTEIN, F. L. 1989. Principal warps: Thin-plate splines and the decomposition of deformations. IEEE Transactions on Pattern Analysis and Machine Intelligence 11: 567-585.

BOOKSTEIN, F. L. 1991. Morphometric tools for landmark data: Geometry and Biology. Cambridge University Press. Cambridge. 
BOOKSTEIN, F. L. 1997. Landmark methods for forms without landmarks: morphometrics of group differences in outline shape. Medical Image Analysis I (3): 225-243.

BORRERO, L. A. 1994-95. Arqueología de la Patagonia. Palimpsesto. Revista de Arqueología id. 4: 9-69.

BORRERO, L. A. y N. V. FRANCO. 2001. Las colecciones líticas del Museo Británico. Anales del Instituto de la Patagonia (Serie Ciencias Humanas) 29: 207-210.

CAHEN, D.; L. H. KEELEY y F. VAN NOTEN. 1979. Stone Tools kits and human behaviour in prehistory. Current Anthropology 20 (4): 661-683.

CARDILLO, M.; I. PÉREZ; N. FRANCO y G. BARRIENTOS. 2005. Variabilidad morfológica en puntas de proyectil pedunculadas del Holoceno en el Sur de Patagonia continental (provincia de Santa Cruz). Trabajo presentado en las Sextas Jornadas de la Arqueología de la Patagonia. Punta Arenas.

CASTRO A. 1989. Aplicación de la tecnología de análisis funcional al estudio de cuarcitas. Revista de estudios regionales 4: 55- 77.

CASTRO A. 1994. El análisis funcional del material lítico por medio de la observación de huellas de uso como alternativa al modelo de clasificación tipológica. Tesis doctoral. Facultad de Ciencias Naturales y Museo, Universidad Nacional de La Plata. La Plata.

CASTRO A. y J. E. MORENO. 1994. Determinación de enmangues en instrumentos líticos por medio de huellas d utilización. Palaeoetnologia 7: 7-20.

CASTRO A. 1996. El análisis funcional de material lítico: un punto de vista. Revista del Museo de La Plata (Nueva Serie) IX: 318-326.

CAVALLI SFORZA, L. L. y M. W. FELDMAN. 1981. Cultural transmission and evolution: a quantitative Approach. Princeton University Press. Princeton.

CHARLIN, J. 2005. Aprovisionamiento de materias primas líticas en el campo volcánico de Pali Aike (Santa Cruz): una primera aproximación a partir del análisis de los núcleos. Werken 7 (2): 39-55.

CHARLIN, J. 2009. Estrategias de aprovisionamiento y utilización de las materias primas líticas en el campo volcánico de Pali Aike (Prov. Santa Cruz). En prensa: British Archaeological Reports, International Series, Archaeopress. Oxford.

CHARLIN, J. y M. CARDILLO. 2009. Morfometría geométrica de los cabezales bifaciales del Campo Volcánico Pali Aike. En preparación.

CHILDS, H. 1997/1936/. El Jimmy. Bandido de la Patagonia. Ediciones de la Universidad de Magallanes, Punta Arenas.
CLOSE, A. 1978. The identification of style in lithic artifacts. World Archaeology 10: 223-237.

CORBELLA, H. 2002. El campo volcánico-tectónico de Pali Aike. En: Geología y recursos naturales de Santa Cruz. Relatorio del XV Congreso Geológico Argentino. El Calafate, 1-18: 285-301. Buenos Aires.

D'ORAZIO, M.; S. AGOSTINI; F. MAZZARINI; F. INNOCENTI; P. MANETTI; M. J. HALLER y A. LAHSEN. 2000. The Pali Aike volcanic Field, Patagonia: Slab-Window Magmatism near the Tip of South America. Tectonophysics 321: 407-427.

FIORE, D. 2006. Poblamiento de imágenes: arte rupestre y colonización de la Patagonia. Variabilidad y ritmos de cambio en tiempo y espacio. En: Tramas en la piedra. Producción y usos del arte rupestre, ed. D. Fiore y M. M. Podestá. Asociación Amigos del Instituto Nacional de Antropología, World Archaeological Congress y Sociedad Argentina de Antropología, pp. 43-62. Buenos Aires.

FOOTE, M. 1993. Contribution of individual taxa to overall morphological disparity. Paleobiology 19: 403-419.

FRANCO, N. V. 1999. Puntas de proyectil y circulación humana: una aproximación tecnológica. En: Libro de Resúmenes del XIII Congreso Nacional de Arqueología Argentina, Universidad Nacional de Córdoba, p. 6. Córdoba.

FRANCO, N. V. 2002. Estrategias de utilización de recursos líticos en la cuenca superior del río Santa Cruz. Tesis doctoral. Facultad de Filosofía y Letras, Universidad de Buenos Aires. Buenos Aires.

FRANCO, N. V. 2004. Rangos de acción, materias primas y núcleos preparados al sur de Lago Argentino. En: Contra Viento y Marea. Arqueología de la Patagonia, M. T. Civalero, P. Fernández y A. G. Guráieb (comps.), pp. 105-116, Instituto Nacional de Antropología y Pensamiento Latinoamericano y Sociedad Argentina de Antropología. Buenos Aires.

FRANCO, N. V. 2008. La estructura tecnológica regional y la comprensión de la movilidad humana: tendencias para la cuenca del río Santa Cruz. En: Arqueología del extremo sur del continente americano. Resultados de nuevos proyectos, capítulo 4, pp. 119-154. Comp. L. A. Borrero y N. V. Franco. Ed. Instituto Multidisciplinario de Historia y Ciencias Humanas (CONICET). Buenos Aires.

FRANCO, N. V. y L. A. BORRERO. 2003. Mind the Gap: Technological Variations between Both sides of the Magellan Strait. En: Abstracts of the Fifth World Archaeological Congress, p. 142. Washington D. C. 
FRANCO, N. V.; M. CARDILlO y L. A. BORRERO. 2005. Una primera aproximación a la variabilidad presente en las puntas de proyectil denominadas "Bird IV". Werken 6: 81-95.

FRANCO, N. V.; C. OTAOLA y M. CARDILLO. 2007. Resultados de los trabajos exploratorios realizados en la margen norte del río Santa Cruz (provincia de Santa Cruz, Argentina). En: Arqueología de Fuego-Patagonia. Levantando piedras, desenterrando huesos... y develando arcanos, pp. 541-553. Ed. F. Morello; M. Martinic; A. Prieto y G. Bahamonde. Ediciones CEQUA. Punta Arenas.

GÓMEZ OTERO, J. 1986-87. Investigaciones arqueológicas en el alero Potrok - Aike (Provincia de Santa Cruz): Una revisión sobre los períodos IV y V de Bird. Relaciones de la Sociedad Argentina de Antropología XVII/1, 173-198.

GÓMEZ OTERO, J. 1987. Posición estratigráfica particular de puntas de los períodos IV y V de Bird en el alero Potrok-Aike (Santa Cruz). En: Primeras Jornadas de Arqueología de la Patagonia, ed. por Dirección de Cultura de la Provincia del Chubut, pp. 125-130. Chubut.

GÓMEZ OTERO, J. 1993. The function of small rockshelters in the Magallanes IV phase settlement system (South Patagonia). Latin American Antiquity 4 (4): 325-345.

HAMMER, Ø.; D. A. HARPER y P. D. RYAN. 2001. Past: Paleontological Statistics Software Package for Education and Data Analysis. Palaeontologia Electrónica 4 (1): 9.

HOWARD, C. 1974. The atlatl: function and performance. American Antiquity 39: 102-104.

IBÁÑEZ ESTÉVEZ J. y J. GONZÁLEZ URQUIJO. 2000. The quantification of use wear polish using image analysis. En: The recent archaeological approaches to the usewear analysis and technical process. The first studies in Honour of S. A. Semenov. Academia Rusa de Ciencias y CIASA - CNRS. San Petersburgo.

KEELEY L. H. 1980. Experimental determination of Stone Tools uses: a Microwear analysis. University of Chicago Press. Chicago.

MANCINI, M. V. 2002. Vegetation and climate during the Holocene in Southwest Patagonia, Argentina. Review of Palaeobotany and Palynology 122: 101-115.

MANCINI, M. V.; M. M. PAEZ; A. R. PRIETO; S. STUTZ; M. TONELLO e I. VILANOVA. 2005. Mid-Holocene climatic variability reconstruction from pollen records (32 $2^{\circ}-52^{\circ} \mathrm{S}$, Argentina). Quaternary International 132: 47-59.
MANSUR-FRANCHOMME, E. 1983. Traces d'utilisation et technologie lithique: examples de la Patagonie. Tesis Doctoral. Universite de Bordeaux. Bordeaux.

- 1987. Outils ethnographiques de la Patagonie. Emmanchement et traces d'utilisation. La main et l'outil: manches et emmanchements préhistoriques. Travaux de la Maison de l'Orient 15: 33-42.

- 1999. Análisis de instrumental lítico: problemas de formación y deformación de rastros de uso. En: Actas del XII Congreso Nacional de Arqueología Argentina, tomo 1: 355-366. La Plata.

MARTUCCI, M. y A. CASTRO. 2009. Análisis funcional de piezas experimentales en dacitas. Manuscrito en preparación.

MASSONE, M. 1979. Panorama etnohistórico y arqueológico de la ocupación Tehuelche y Prototehuelche en la costa del Estrecho de Magallanes. Anales del Instituto de la Patagonia, Serie Ciencias Humanas 10: 69-107.

MASSONE, M. 1989-1990. Investigaciones arqueológicas en la Laguna Thomas Gould. Anales del Instituto de la Patagonia, Serie Ciencias Humanas 19: 87-99.

MENGONI GOÑALONS, G. L. 1999. Cazadores de guanacos de la estepa patagónica. Sociedad Argentina de Antropología. Colección Tesis Doctorales, Buenos Aires.

NAMI, H. 1984a. Análisis tipológico de los instrumentos provenientes del sitio "El Volcán" C. 4. Cuenca del Río Chico, Provincia de Santa Cruz. PREP: Informes de Investigación 1: 55-81.

- 1984b. Algunas observaciones sobre la manufactura de las puntas de proyectil de El Volcán. PREP: Informes de Investigación 1:85-107.

- 1986. Experimentos para el estudio de la tecnología bifacial de las ocupaciones tardías en el extremo sur de Patagonia Continental. PREP: Informes de Investigación 5:1-120.

- 2003. Experimentos para explorar la secuencia de reducción Fell de la Patagonia Austral. Magallania 31:107-138.

NELSON, M. 1991. The Study of Technological Organization. Archaeological Method and Theory 3: 57-100.

ODELL, G. y F. COWAN. 1986. Experiments with spears and arrows on animal targets. Journal of Field Archaeology 13 (2): 195-212.

ORQUERA, L. A. 1987. Advances in the archaeology of the Pampa and Patagonia. Journal of World Archaeology I: 333-413.

PRIETO, A. 1989-90. Cazadores tardíos en la zona fronteriza del paralelo $52^{\circ}$ sur. El alero Peggy Bird. Anales del Instituto de la Patagonia Serie Cs. Sc. 19: 73-85. 
RAPOPPORT, E. H.; A. LADIO y E. A. SANZ. 2003. Plantas nativas comestibles de la Patagonia andina argentinachilena, parte II. Programa de Extensión Universitaria, Universidad Nacional del Comahue, Departamento de Ecología, Centro Regional Universitario de Bariloche. San Carlos de Bariloche.

RATTO, N. 1988. Proyectiles en acción. En: Precirculados del IX Congreso Nacional de Arqueología Argentina, Instituto de Ciencias Antropológicas, Facultad de Filosofía y Letras, Universidad de Buenos Aires, pp. 6-19. Buenos Aires.

- 1993. What and How did they Hunt? Methodological Essay to Approach the Question of Prehistoric Hunting Tecniques. En: Explotación de Recursos Faunísticos en Sistemas Adaptativos Americanos, Lanata, J. L. (Comp.), Arqueología Contemporánea 4, edición especial, pp. 135-148. Buenos Aires.

- 1994. Funcionalidad vs. adscripción cultural: cabezales líticos de la margen norte del Estrecho de Magallanes. En: Arqueología de cazadores-recolectores. Límites, casos y aperturas, Lanata, J. L. y Borrero, L. A. (Ed.), Arqueología Contemporánea 5 , edición especial, pp. 105-120. Buenos Aires.

REYES, M. y A. CASTRO. 2004 Análisis microscópico de rastros de uso en instrumentos de basalto: factibilidad, potencialidad y limitaciones. Trabajo presentado al XV Congreso Nacional de Arqueología Argentina. Rio Cuarto 20 al 25 de setiembre 2004. Córdoba.

ROHLF, F. J., 1990. Morphometrics. Annual Review of Ecology and Systematics 21: 299-316.

- 1993. Relative warp analysis and an example of its application to mosquito wings in Marcus, L. F., Bello, E., Garcia-Valdecasas, A (Ed.), Contributions to Morphometrics. Museo Nacional de Ciencias Naturales (CSIC), Madrid, Spain, 8:131-159.

- 2004. TPS Series Software. Disponible en: http//life. bio.sunysb.edu/morph/.

ROTS V. 2003. Towards an Understanding of hafting: the macro and microscopic evidence. Antiquity 77 (298): 805-815.
- 2005. Hafting traces on flint tools: possibilities and limitations of macro and microscopic approaches. En: Prehistoric Technology 40 years later: Functional studies and the Russian Legacy. Book of abstracts, pp. 31-32. Verona.

SANGUINETTI DE BÓRMIDA, A. 1984. Noticias sobre el sitio "El Volcán", su relación con el poblamiento tardío de las cuencas de los ríos Gallegos y Chico (Provincia de Santa Cruz, Argentina). PREP: Informes de Investigación 1: 5-34.

SOKAL R., R y F, J ROHLF. 1979. Biometría. Principios y métodos estadísticos en la investigación biológica. Blume. Madrid.

STERN, CH. 2000. Sources of obsidian artefacts from the Pali Aike, Fell's Cave and Cañadón La Leona archaeological sites in southernmost Patagonia. Desde el país de los gigantes. Perspectivas arqueológicas en Patagonia, (UNPA ed.), Tomo II, Río Gallegos: 43-55.

TOSELLI, A.; J. PIJOAN-LOPEZ; J. A. BARCELÓ; I. VILA y A. MITRA. 2005. Kinematics and use-wear traces: an attempt of characterisation through image digitalisation. En: Prehistoric Technology 40 years later: Functional studies and the Russian Legacy. Book of abstracts, pp. 26-27. Verona.

TRINGHAM, R.; G. COOPER; G. ODELL; A. VOYTEK y A. WHITMAN. 1974. Experimentation in the formation of edge damage: a new approach to lithic analysis. Journal of Field Archaeology 1:171-196.

VAUGHAN, P. 1985. Use wear of flaked stone tools. PhD. Dissertation. University of Arizona Press. Arizona.

VIGNATI, M. A. 1934. Resultados de una excursión por la margen sur del río Santa Cruz. Notas Preliminares del Museo de La Plata 2: 77-151.

WIESSNER, P. 1983. Style and social information in Kalahari San projectil points. American Antiquity 48 (2): 253276.

ZELDITCH, M. L.; D. L. SWIDERSKI; H. D. SHEETS y W. L. FINK. 2004. Geometric Morphometric for Biologists: A Primer. Academic Press. London. 